Article

\title{
DFT Calculations of the Structural, Mechanical, and Electronic Properties of TiV Alloy Under High Pressure
}

\author{
Fang $\mathrm{Yu}^{1}$ and $\mathrm{Yu} \mathrm{Liu}{ }^{2, *(D)}$ \\ 1 School of Software and Communication Engineering, Xiangnan University, Chenzhou 423000, China \\ 2 State Key Laboratory of Advanced Design and Manufacturing for Vehicle Body, Hunan University, \\ Changsha 410082, China \\ * Correspondence: lyu1006@hnu.edu.cn
}

Received: 8 July 2019; Accepted: 29 July 2019; Published: 1 August 2019

check for updates

\begin{abstract}
A calculation program based on the density functional theory (DFT) is applied to study the structural, mechanical, and electronic properties of TiV alloys with symmetric structure under high pressure. We calculate the dimensionless ratio, elastic constants, shear modulus, Young's modulus, bulk modulus, ductile-brittle transition, material anisotropy, and Poisson's ratio as functions of applied pressure. Results suggest that the critical pressure of structural phase transition is $42.05 \mathrm{GPa}$ for the TiV alloy, and structural phase transition occurs when the applied pressure exceeds $42.05 \mathrm{GPa}$. High pressure can improve resistance to volume change, as well as the ductility and atomic bonding, but the strongest resistances to elastic and shear deformation occur at $P=5 \mathrm{GPa}$ for TiV alloy. Furthermore, the results of the density of states (DOS) indicate that the TiV alloy presents metallicity. High pressure disrupts the structural stability of the TiV alloy with symmetry, thereby inducing structural phase transition.
\end{abstract}

Keywords: TiV alloy; Symmetric structure; DFT calculation; mechanical property; elastic constant; electronic structure; applied pressure

\section{Introduction}

TiV alloys with high gravimetric and volumetric hydrogen storage capacities have been widely regarded as important hydrogen storage materials [1-4]. Iba and Akiba [5] reported TiV-Mn alloys with multiphase nanostructures of body-centered cubic (BCC) and C14-type Laves phases, which had a large hydrogen capacity and excellent desorbing properties. Then, they revealed that the improved hydrogen sorption properties were attributed to the generation of multiphase nanostructures. Yu et al. [6] studied the hydrogen storage performance of a single BCC phase Ti-40V-10Cr-10Mn alloy, and results indicated that the largest hydrogen absorption capacity of the Ti-V-Cr-Mn alloy can reach $4.2 \mathrm{wt} \%$, exceeding that of other hydrogen storage alloys, such as rare-earth (RE)-, Ti-, and Zr-based alloys. Nomura and Akiba [7] also investigated 26 types of alloys composed of $\mathrm{Ti}(33-47 \mathrm{~mol} \%)-\mathrm{V}(42-67 \mathrm{~mol} \%)-\mathrm{Fe}(0-14 \mathrm{~mol} \%)$, and then found that the most suitable proportion for hydrogen absorption was $\mathrm{Ti}_{43.5} \mathrm{~V}_{49.0} \mathrm{Fe}_{7.5}$, and the hydrogen capacity reached up to $3.90 \mathrm{wt} \%(\mathrm{H} / \mathrm{M}$ $=1.90$ ) at $253 \mathrm{~K}$. Meanwhile, Seo et al. [8] reported that the $\mathrm{V}_{0.68} \mathrm{Ti}_{0.20} \mathrm{Fe}_{0.12}$ alloy showed the largest hydrogen capacity of $3.6 \mathrm{wt} \%$, and heat treatment can effectively improve hydrogen capacity, such as that in the $\mathrm{V}_{0.375} \mathrm{Ti}_{0.20} \mathrm{Cr}_{0.30} \mathrm{Mn}_{0.075}$ alloy with a hydrogen capacity of $2.2 \mathrm{wt} \%$. Therefore, as the new hydrogen storage materials, TiV-based alloys have been greatly investigated [4,9-11]. However, the structural, mechanical, and electronic properties of TiV alloys remain unrevealed under high pressure due to the complexity of calculations, thereby limiting the applications of $\mathrm{TiV}$ alloys under high pressure. 
Thus, this work uses first-principle calculations to study the structural, mechanical, and electronic properties of the TiV alloy with symmetry systematically within the frame of the density functional theory (DFT) and computes in detail the some variables with respect to the different pressures, such as the dimensionless ratio, elastic constants, elastic modulus, ductile-brittle transition, anisotropy factors, and Poisson's ratio, along with electronic properties. Moreover, the calculated results agree well with other experimental data and theoretical results. Therefore, the results can provide valuable guidance for the development and application of TiV alloys in the area of hydrogen storage materials.

\section{Methodology}

In the present work, all DFT calculations were performed by the Cambridge Serial Total Energy Package Program [12-14], which aims to obtain the electronic and energy properties of each structure in TiV alloys. The exchange-correlation function was decided by the generalized gradient approximation of Perdew-Burke-Ernzerhof [15]. The electronic states of Ti $\left(4 s^{2} 3 p^{6} 3 d^{2}\right)$ and V $\left(4 s^{2} 3 p^{6} 3 d^{3}\right)$ are the valence electrons, and the Vanderbilt-type ultrasoft pseudopotentials were applied to address the ion-electron interactions [16]. On the basis of a precise convergence test, the plane-wave cutoff energy was optimized as $400 \mathrm{eV}$, and the Brillouin-zone $k$-point grid [17] was selected as $13 \times 13 \times 13$ in the electronic calculations of the TiV alloy. The space group of the TiV alloy belongs to $I m-3 m$, and the symmetric crystal structure of the TiV alloy is shown in Figure 1, and the Broyden-Fletcher-Goldfarb-Shanno (BFGS) algorithm [18] was applied in the process of structural geometry optimization with respect to the applied pressures ranging from -10 to $50 \mathrm{GPa}$. The energy convergence criterion was set at $1.0 \times 10^{-6} \mathrm{eV} /$ atom in self-consistent calculations, and the Hellmann-Feynman force of each atom was lower than $0.01 \mathrm{eV} / \AA$.

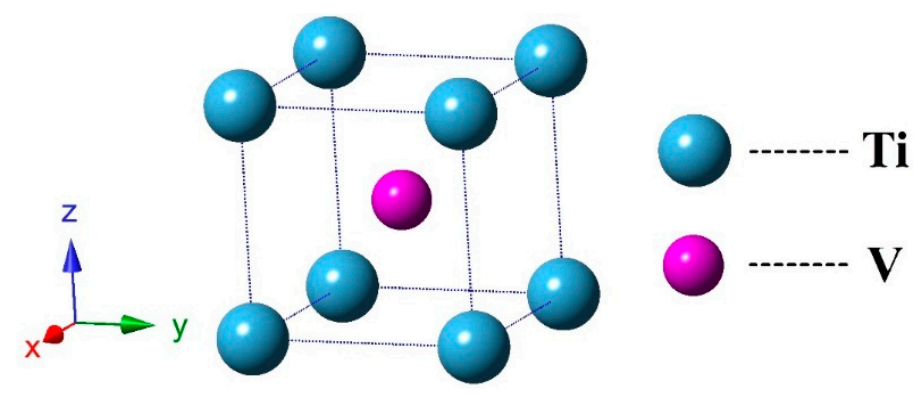

Figure 1. Symmetric Crystal structure of the TiV alloy.

\section{Results and Discussion}

\subsection{Structural Properties and Stability}

Prior to studying the various properties of the TiV alloy, the most stable crystal structure of the TiV alloy was first obtained, and the $E-V$ data of total energy $E$ with respect to volume $V$ were calculated, and then they were accurately fitted by the Birch-Murnaghan equation of state [19], as shown in Figure 2, where the variation ranges of volume $V$ are from $0.9 V_{0}$ to $1.1 V_{0}$. During the geometry optimization of TiV alloys, the full optimization for a unit cell was implemented to obtain the total energy of the stable state. Figure 2 shows that for volume $V=V_{0}\left(V_{0}=29.971 \AA^{3}\right)$, the total energy reached the minimum value $\left(E_{t}=-16.457 \mathrm{eV}\right)$, and the lattice constant was accordingly $a_{0}=3.107 \AA$, indicating the structural parameters of the TiV alloy under the most stable structure, where $V_{0}$ is the primitive cell volume, and $a_{0}$ is the equilibrium lattice constant at pressure $P=0$ and temperature $T=0$, respectively. The present results agree well with other approaches $[10,20-25]$ and are comparatively listed in Table 1. 


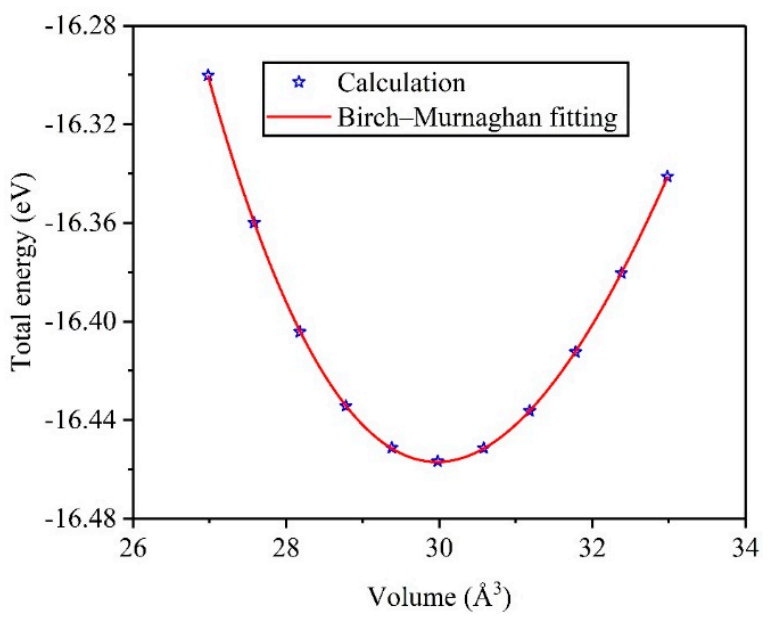

Figure 2. The $E-V$ curve of total energy $E$ with respect to cell volume $V$ of the TiV alloy.

Table 1. Comparisons of the calculated lattice constant to the ones of others for TiV alloy.

\begin{tabular}{ccl}
\hline TiV Alloy & This Work & \multicolumn{1}{c}{ Others } \\
\hline & & $3.156[10], 3.163[20], 3.165[21]$ \\
Lattice constant $a(\AA)$ & 3.107 & $3.120[22], 3.159[23], 3.140[24]$ \\
& & $3.280[25]$ \\
\hline
\end{tabular}

To investigate the dependencies of the lattice constant and unit cell volume on the applied pressure, a series of structural optimizations were conducted to obtain the corresponding lattice constant under various applied pressures. Then, the dimensionless ratios $a / a_{0}$ and $V / V_{0}$ with respect to the different applied pressures, which are depicted in Figure 3, decreased monotonously with an increment of applied pressure, and the compression ratio of the volume was much larger compared with the lattice constant at the same applied pressure, thereby indicating that high pressure greatly reduces interatomic distance and leads to strong electron interactions.

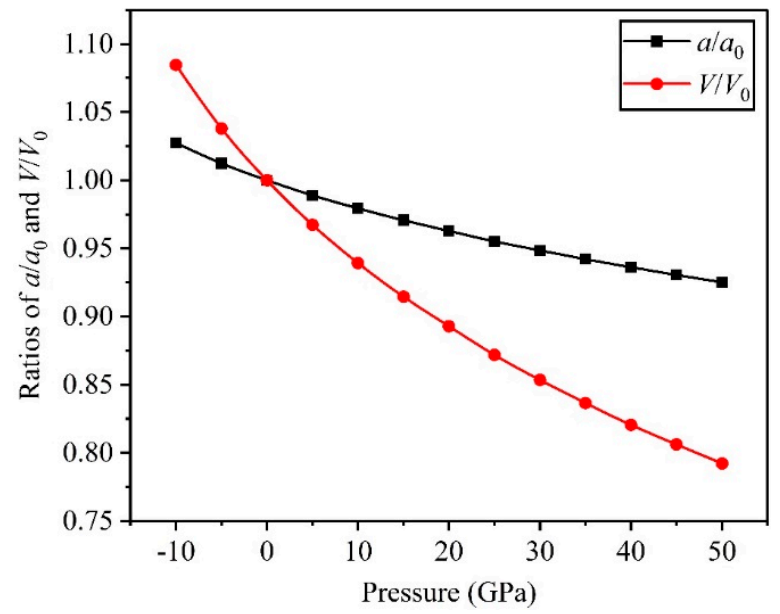

Figure 3. Dependencies of dimensionless ratios $a / a_{0}$ and $V / V_{0}$ on the applied pressure for the TiV alloy.

In Figure 3, we fit the dependencies of dimensionless ratios $a / a_{0}$ and $V / V_{0}$ as a function of the applied pressure by using quadratic polynomial, and they are expressed as follows:

$$
\begin{aligned}
& a / a_{0}=1.001-2.240 \times 10^{-3} P+1.501 \times 10^{-5} P^{2} \\
& V / V_{0}=1.005-6.690 \times 10^{-3} P+5.087 \times 10^{-5} P^{2}
\end{aligned}
$$


In the anisotropic material, the elastic constants, which denote the ability to resist applied stress, are considered important physical quantities in measuring the structural stability of a material. For cubic crystals, the elastic constants include three coefficients, namely, $C_{11}, C_{12}$, and $C_{44}$. In accordance with the stability criterion [26,27], three elastic constants of cubic crystals fit with the following expressions:

$$
\left(C_{11}-C_{12}\right)>0, C_{11}>0, C_{44}>0,\left(C_{11}+2 C_{12}\right)>0
$$

At $P=0$ and $T=0$, Table 2 lists the calculated elastic constants, bulk modulus $B$, Young's modulus $E$, and shear modulus $G$, as well as Poisson's ratio $\sigma$. Table 2 shows that the calculated results are consistent with the work of Ikehata et al. [25].

Table 2. Comparisons of the calculated results with the work of Ikehata et al. [25] at $P=0$ and $T=0$.

\begin{tabular}{ccc}
\hline TiV Alloy & This Work & Ikehata et al. [25] \\
\hline$C_{11}(\mathrm{GPa})$ & 178.16 & 169.6 \\
$C_{12}(\mathrm{GPa})$ & 126.92 & 122.3 \\
$C_{44}(\mathrm{GPa})$ & 21.52 & 33.6 \\
$C_{44}(\mathrm{GPa})$ & 144.00 & 138.07 \\
Young's modulus $E(\mathrm{GPa})$ & 65.71 & 81.81 \\
Shear modulus $G(\mathrm{GPa})$ & 23.07 & 29.19 \\
Poisson's ratio $\sigma$ & 0.42 & 0.40 \\
\hline
\end{tabular}

Figure 4 demonstrates the dependencies of elastic constants $C_{11}, C_{12}$, and $C_{44}$ on the applied pressure for the TiV alloy, revealing that for the pressure $P$ changing from $0 \mathrm{GPa}$ to $42.05 \mathrm{GPa}, C_{11}$ and $C_{12}$ increase gradually, but the elastic coefficient $C_{44}$ decreases. Furthermore, the elastic coefficient $\mathrm{C}_{44}$ reduces slowly to zero with increasing applied pressure and then dramatically transforms into a negative value under the high pressure exceeding $42.05 \mathrm{GPa}$, which is unfit for the stability criterion of Equation (3). Thus, a high pressure exceeding $42.05 \mathrm{GPa}$ disrupts the crystalline structure of the $\mathrm{TiV}$ alloy, namely, the TiV alloy is inclined to induce structural phase transition in the case of a high pressure of $42.05 \mathrm{GPa}$.

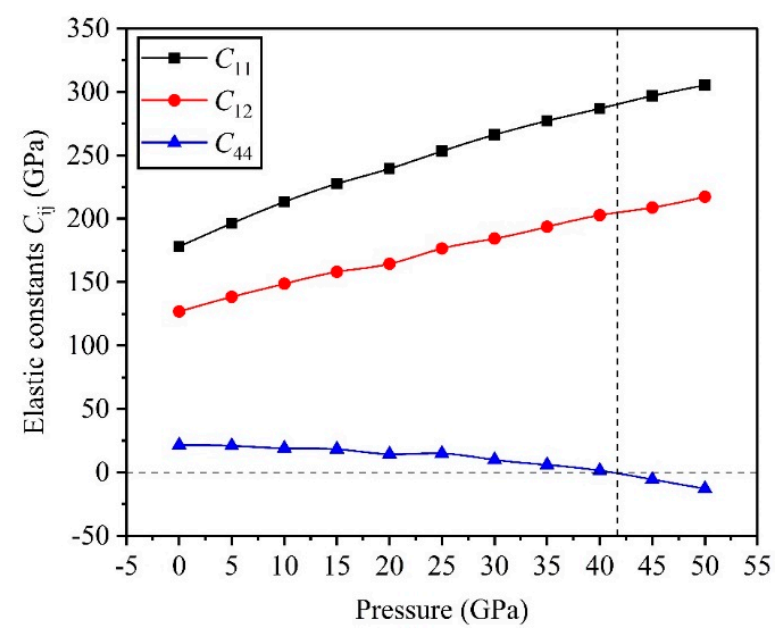

Figure 4. Dependencies of the elastic constants $C_{i j}$ on the applied pressure for the TiV alloy.

\subsection{Mechanical Properties}

The mechanical properties of materials, such as high strength, good plasticity, and excellent deformation resistance, are closely related to material moduli, like shear modulus $G$, Young's modulus $E$, and bulk modulus $B[28,29]$. A large modulus has great resistance to material deformation. 
Furthermore, we can apply Equations (4)-(6) [30] to calculate the modulus values of these materials. These modulus values depend on the calculated elastic constants, as listed in Table 2:

$$
\begin{gathered}
B=\frac{1}{3}\left(C_{11}+2 C_{12}\right) \\
G=\frac{1}{2}\left(G_{V}+G_{R}\right) \\
E=\frac{9 B G}{3 B+G}
\end{gathered}
$$

where $G_{V}=\left(C_{11}-C_{12}+3 C_{44}\right) / 5$ and $G_{R}=5\left(C_{11}-C_{12}\right) C_{44} /\left[4 C_{44}+3\left(C_{11}-C_{12}\right)\right]$, and $G_{V}$ and $G_{R}$ denote the Voigt and Reuss shear moduli, respectively.

Figure 5 demonstrates the dependencies of shear modulus $G$, Young's modulus $E$, and bulk modulus $B$ on the applied pressure. Prior to structural phase transition, the bulk modulus $B$ increased with increasing applied pressure, indicating that the high pressure leads to a strong resistance to volume change for the TiV alloy, and the maximum bulk modulus $B_{\max }$ is equal to $233.90 \mathrm{GPa}$ at $P=42.05 \mathrm{GPa}$. However, shear modulus $G$ and Young's modulus $E$ initially increased from $0 \mathrm{GPa}$ to $5 \mathrm{GPa}$ and then decrease slowly with increasing applied pressure. The maximum values of Young's modulus $E$ and shear modulus $G$ were 68.47 and $23.98 \mathrm{GPa}$ at $P=5 \mathrm{GPa}$, respectively, implying that the applied pressure at this value generates the maximum resistance to elastic and shear deformations for the TiV alloy.

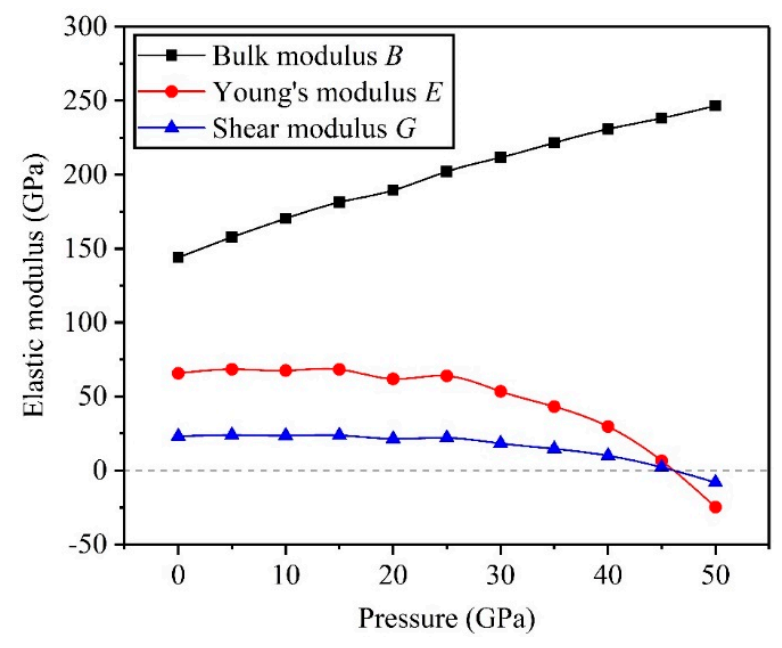

Figure 5. Dependencies of shear modulus $G$, Young's modulus $E$, and bulk modulus $B$ on the applied pressure for the TiV alloy.

To study the structural phase transition of materials, the present work aimed to analyze the ductile-brittle transition under different pressures. Pugh [28] raised that the modulus ratio $B / G$ can be considered the key physical quantities in measuring the ductile/brittle properties for polycrystalline materials. Generally, 1.75 is the critical value of the ratio $B / G$ for distinguishing ductile and brittle materials. The materials are characterized for the ductility by $B / G>1.75$, and they are characterized for the brittleness by $B / G<1.75$. Moreover, the ductility of materials increases with increasing $B / G$ ratio. Otherwise, the brittleness increases. These conditions are equally suitable for investigating the ductile-brittle transition of intermetallic compounds [31]. Figure 6 exhibits the calculated results of the present work, showing that the modulus ratio $B / G$ is approximately 6.24 under $P=0 \mathrm{GPa}$, and the $B / G$ ratio (> 1.75) increases with an increase of applied pressure. Thus, the results from Figure 6 indicate that the TiV alloy is essentially characterized by excellent ductility, and high pressure can improve the ductility of the TiV alloy. 


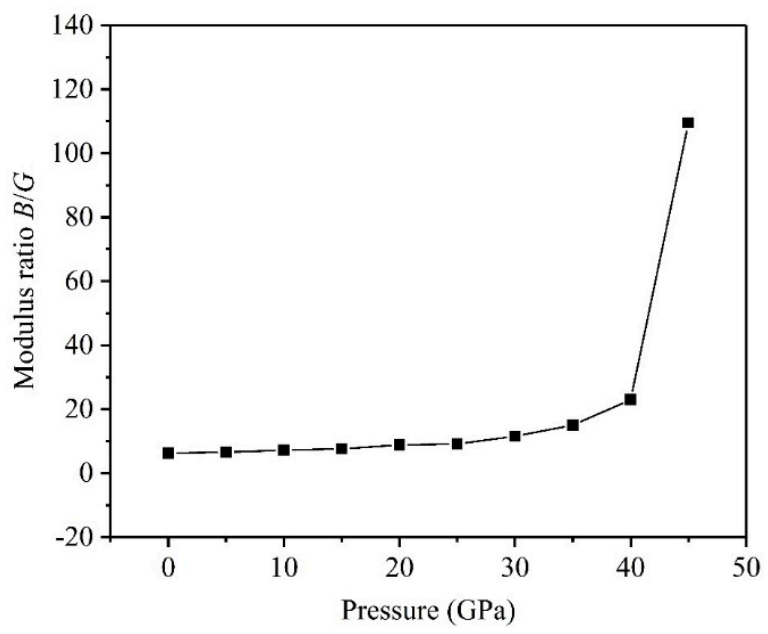

Figure 6. Dependencies of the modulus ratio $B / G$ on the applied pressure for the TiV alloy.

\subsection{Anisotropy}

For anisotropic materials, we used elastic anisotropy to study their mechanical properties. The anisotropy factor $A$ plays a key role in measuring the elastic anisotropy of materials, which are characterized by the isotropy at $A=1$. Otherwise, they are characterized by the anisotropy at $A \neq 1$. Various anisotropy factors $A(A \neq 1)$ correspond to the varying degrees of material anisotropy, and the material anisotropy is stronger when the anisotropy factors $A$ are more deviated [32,33]. In the light of the work of Yoo [34], the cross-slip pinning model was presented to investigate the cross-slip pinning process of screw dislocations. The large factor $A$ can increase the driving force of screw dislocation motion, and then promote the cross-slip pinning process of such dislocations. Here, we calculated the anisotropy factors $A_{(100)[001]}$ and $A_{(110)[001]}$ using Equations (7) and (8), respectively. They are expressed by the three elastic constants of cubic crystals, as follows [35,36]:

$$
\begin{gathered}
A_{(100)[001]}=\frac{2 C_{44}}{C_{11}-C_{12}} \\
A_{(110)[001]}=\frac{C_{44}\left(C^{\prime}+2 C_{12}+C_{11}\right)}{C_{11} C^{\prime}-C_{12}{ }^{2}}
\end{gathered}
$$

where $C^{\prime}=C_{44}+\left(C_{11}+C_{12}\right) / 2, A_{(100)[001]}$ is the anisotropy factor in the (100)[001] direction, and $A_{(110)[001]}$ in the (110)[001] directions. Based on the calculations of Equations (7) and (8), Figure 7 shows the dependencies of the two anisotropy factors on the applied pressure. These factors helped investigate the mechanical properties of the TiV alloy under various pressures. Results in Figure 7 indicate that the anisotropy factors of the TiV alloy are $A_{(100)[001]}=0.840$ and $A_{(110)[001]}=0.875$ at $P=0 \mathrm{GPa}$, suggesting that the TiV alloy is essentially characterized by the anisotropy. As the applied pressure increases, the anisotropy factors $A_{(100)[001]}$ and $A_{(110)[001]}$ decrease rapidly, indicating that a larger applied pressure leads to a stronger anisotropy for the TiV alloy. Moreover, the cross-slip process of screw dislocations can be accelerated due to the strong anisotropy caused by the high pressure. 


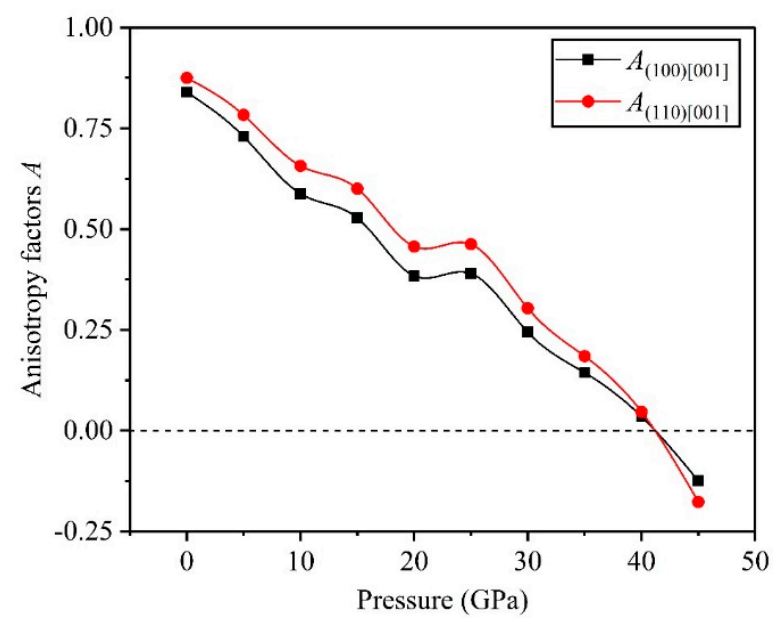

Figure 7. Dependencies of anisotropy factors on the applied pressure for the TiV alloy.

To investigate the plastic property of the TiV alloy under different pressures, we introduced the Poisson's ratio $\sigma$. Generally, the range of Poisson's ratio $\sigma$ is between -1 and 0.5 , and the material has better plasticity when Poisson's ratio $\sigma$ is larger. Based on the key factors of atomic scale, Poisson's ratio $\sigma$ mainly depended on the type of interatomic bonding [36]. In the work of Reed and Clark [37], Poisson's ratios $\sigma_{\min }=0.25$ and $\sigma_{\max }=0.5$ were identified as the minimum and maximum values in investigating central force solids, respectively. Herein, we define the $\sigma_{[001]}$ and $\sigma_{[111]}$ as the Poisson's ratios in the directions of [001] and [111], respectively, and they were obtained using Equations (9) and (10) [36,38]. Figure 8 depicts the dependencies of Poisson's ratios $\sigma_{[001]}$ and $\sigma_{[111]}$ on the applied pressure. We can obtain the values of $\sigma_{[001]}=0.416$ and $\sigma_{[111]}=0.429$ at $P=0 \mathrm{GPa}$. Thus, for the $\mathrm{TiV}$ alloy in nature, interatomic bonding is mainly characterized by the central force in the directions of [001] and [111]. As the applied pressure increases, the value of $\sigma_{[001]}$ initially decreases and then increases, but that of $\sigma_{[111]}$ gradually increases and then tends to achieve the maximum value of $\sigma_{\max }=0.5$, thereby indicating that the high pressure enhances the central force and plasticity of the $\mathrm{TiV}$ alloy in the [111] uniaxial direction.

$$
\begin{gathered}
\sigma_{[001]}=\frac{C_{12}}{C_{11}+C_{12}} \\
\sigma_{[111]}=\frac{C_{11}+2 C_{12}-2 C_{44}}{2\left(C_{11}+2 C_{12}+C_{44}\right)}
\end{gathered}
$$

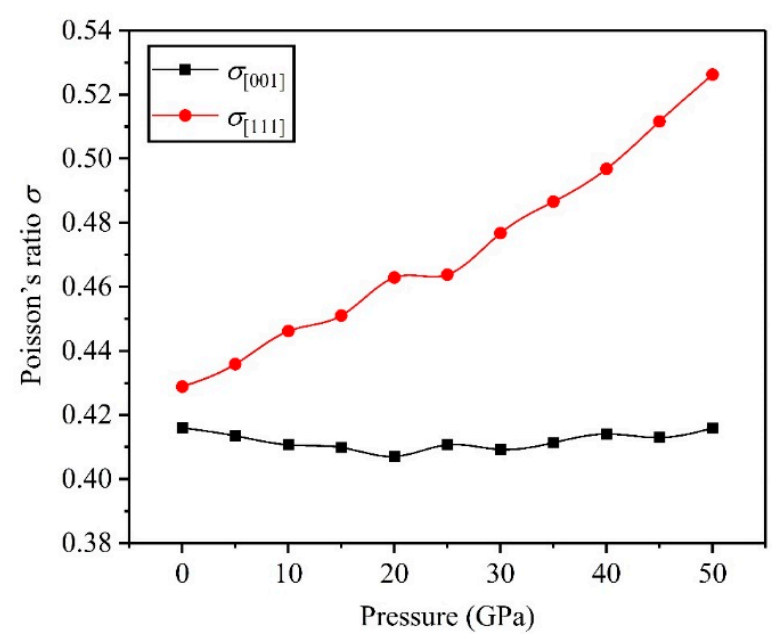

Figure 8. Dependencies of Poisson's ratios on the applied pressure for the TiV alloy. 
The mechanical properties of the materials can also be evaluated by other physical quantities. The evaluation provides valuable guidance for studying material strength and deformation. Variables $G_{(100)[010]}$ and $G_{(110)[1 \overline{1} 0]}$ are the shear moduli in the (100)[010] and (110)[1 $\left.\overline{10}\right]$ directions, respectively, where they are expressed by $G_{(100)[010]}=C_{44}$ and $G_{(110)[1 \overline{1} 0]}=\left(C_{11}-C_{12}\right) / 2$. Similarly, variable $E_{\langle 100\rangle}$ is the Young's modulus in the $\langle 100\rangle$ uniaxial directions, and $E_{\langle 100\rangle}=\left(C_{11}-C_{12}\right)\left[1+C_{12} /\left(C_{11}+C_{12}\right)\right][33$, 39]. According to the definitions of these moduli, Figure 9 exhibits their dependencies on the applied pressure. Results show that shear modulus $G_{(110)[1 \overline{10}]}$ is always greater than $G_{(100)[010]}$ under any pressure, thereby indicating that the (110) $[1 \overline{1} 0]$ direction has stronger resistance to shear deformation compared with the (100)[010] direction. Moreover, $G_{(110)[1 \overline{1} 0]}$ increases gradually with increasing applied pressure, thereby revealing that the high pressure enhances the resistance to shear deformation in the (110) [110] direction. However, the high pressure reduces the resistance to shear deformation in the (100) [010] direction due to the decrement of $G_{(100)[010]}$. Young's modulus $E_{\langle 100\rangle}$ increases gradually with increasing applied pressure. Thus, high pressure improves the resistance to elastic deformation in the $\langle 100\rangle$ directions. As another physical quantity, the Cauchy pressure $C_{12}-C_{44}$ is generally used to reflect the characteristic of atomic bonding, and it can reveal the nature of the bonding from the atomic scale [40]. A positive Cauchy pressure denotes that the atomic bonding exhibits metallic properties. Thus, a homogeneous electron gas exists near the spherical atoms, and the electron distribution shows the same characteristics without regionality and directionality. However, a negative Cauchy pressure is characterized by the directional bonding, and the directional characteristic increases with increasing Cauchy pressure $[33,41]$. Results in Figure 9 show that the value of Cauchy pressures $C_{12}-C_{44}$ is always positive under any pressure and increases linearly with increasing applied pressure, thereby suggesting that the atomic bonding of the TiV alloy is mainly the metallic bond, and high pressure leads to a strong atomic bonding.

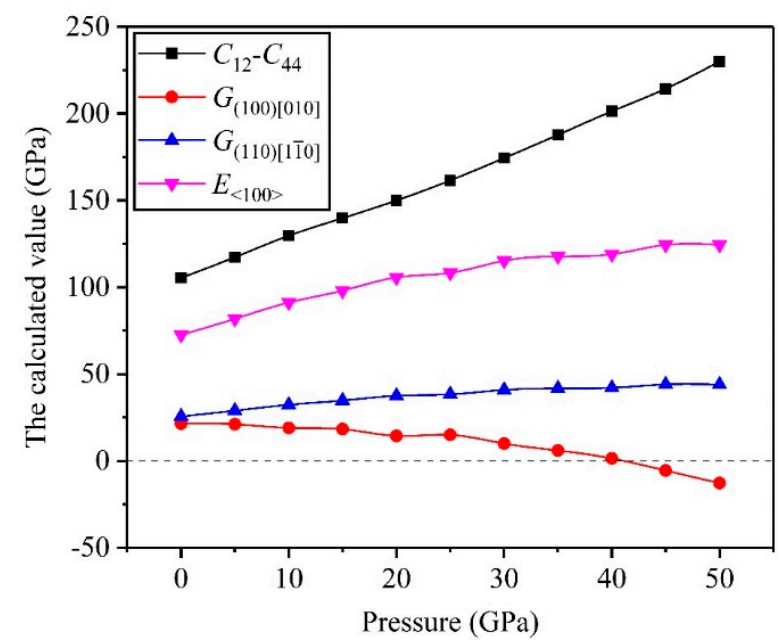

Figure 9. Dependencies of material moduli on the applied pressure for the TiV alloy.

\subsection{Electronic Properties}

To further investigate the bonding mechanisms of the TiV alloy, we obtained the electronic structures to reveal the stability changes of the TiV alloy under different pressures. Figure 10 depicts the partial density of states (PDOS) and total DOS (TDOS) of the TiV alloy under $P=0 \mathrm{GPa}$, and the Fermi level $\left(E_{\mathrm{F}}=0 \mathrm{eV}\right)$ is marked by the red dash line. The value of TDOS at $E_{\mathrm{F}}$ was not equal to zero, indicating that the $\mathrm{TiV}$ alloy presents the metallicity in nature and agrees well with the results in Figure 9. In light of the PDOS of the TiV alloy, the contributions to DOS are mainly obtained from the Ti-3d and V-3d states at the $E_{\mathrm{F}}$, and the Ti-4s and V-4s states have no contributions to DOS. 


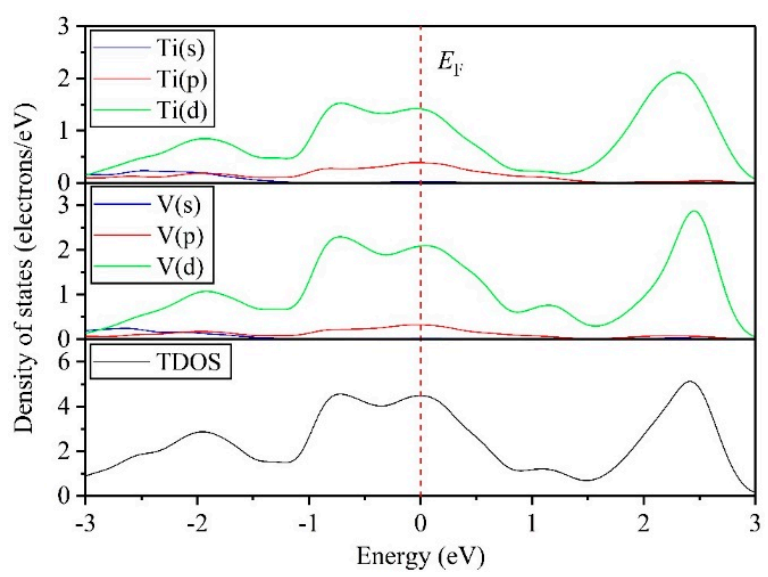

Figure 10. PDOS and TDOS for the TiV alloy under $P=0 \mathrm{GPa}$.

Simultaneously, we also investigated the influences of the applied pressure on TDOS of the TiV alloy, and Figure 11 demonstrates the dependencies of the TDOS of the TiV alloy on the applied pressure. With increasing applied pressure, the valence band energy increases but the conduction band energy decreases, thereby resulting in the decrement of the band gap between valence band and conduction band. Then, the stability of the crystal structure decreases to a certain extent. The crystal structure of the TiV alloy easily produces the structural phase transition under high pressure. Results agree well with the previous results.

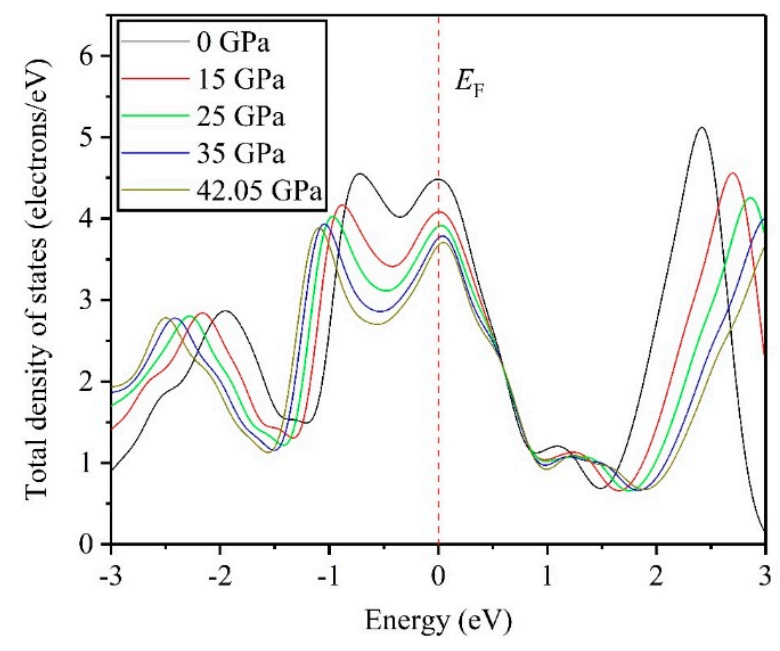

Figure 11. TDOS for the TiV alloy under various pressures.

Figure 12 plots the schematic of the isosurface contours of charge density under different pressures. The figure intuitively reflects the local chemical bonding between Ti and V atoms. Figure 12 shows that the local charge density between Ti and V atoms increases gradually with the increase of applied pressure, suggesting that the electron interaction between Ti and $\mathrm{V}$ atoms becomes increasingly strong under high pressure. Meanwhile, the results indirectly reflect that the local chemical bonding combined with the electrons and ions, between Ti and $\mathrm{V}$ atoms becomes stronger with increasing the applied pressure as well. The high pressure induces strong interactions between the Ti and V atoms, leading to structural instability under high pressure. Thus, the high pressure tends to destroy the structural stability of the TiV alloy, thereby inducing structural phase transition. 

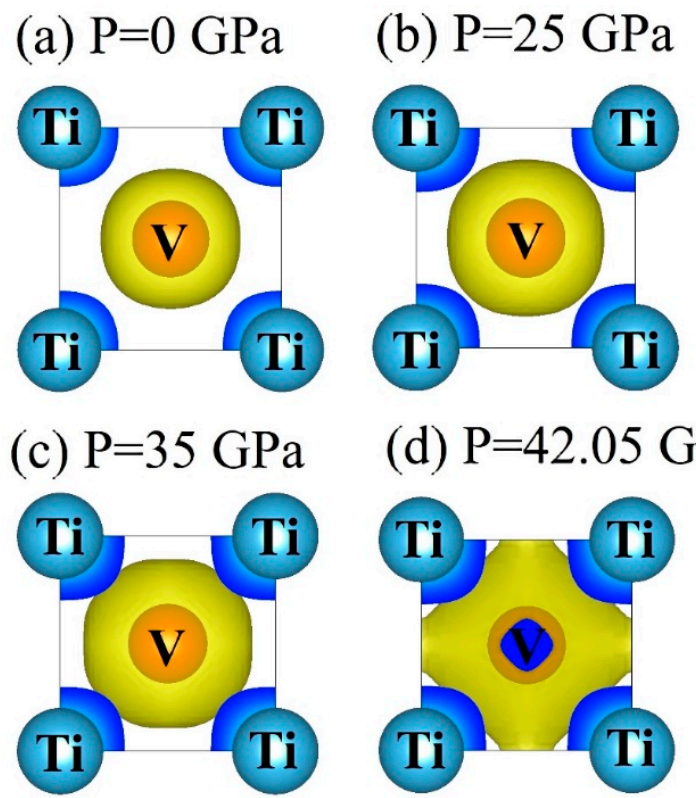

(d) $\mathrm{P}=42.05 \mathrm{GPa}$

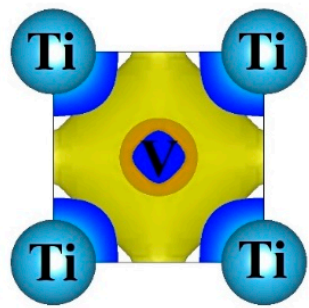

Figure 12. Schematic of isosurface contours of charge density for the TiV alloy under different pressures. The isosurface levels are set as $0.0406 r_{0}^{-3}\left(r_{0}\right.$ : Bohr radius).

\section{Conclusions}

(1) The critical pressure of the structural phase transition for the TiV alloy is $42.05 \mathrm{GPa}$, and the symmetric crystal structure of the TiV alloy produces structural phase transition when the applied pressure exceeds $42.05 \mathrm{GPa}$.

(2) The high pressure can improve resistance to volume change, but the biggest resistances to elastic and shear deformation occur under $P=5 \mathrm{GPa}$ for the TiV alloy.

(3) The results of Pugh's B / $G$ ratio suggest that the TiV alloy is essentially characterized by excellent ductility, and high pressure can enhance the ductility of the TiV alloy.

(4) The (110) $[1 \overline{1} 0]$ direction has stronger resistance to shear deformation than the (100) [010] direction, and high pressure improves resistance to elastic deformation in the $\langle 100\rangle$ direction. Cauchy pressure reveals that the atomic bonding of the TiV alloy is mainly the metallic bond, and high pressure leads to strong atomic bonding.

(5) DOS results indicate that the TiV alloy presents metallicity, and high pressure disrupts the structural stability of the TiV alloy, thereby inducing structural phase transition.

Author Contributions: Conceptualization, F.Y. and Y.L.; methodology, F.Y. and Y.L.; software, F.Y.; validation, F.Y. and Y.L.; formal analysis, F.Y. and Y.L.; investigation, F.Y. and Y.L.; resources, F.Y.; data curation, F.Y. and Y.L.; writing-original draft preparation, F.Y. and Y.L.; writing-review and editing, F.Y. and Y.L.; visualization, F.Y.; supervision, F.Y. and Y.L.; project administration, F.Y. and Y.L.; funding acquisition, F.Y.

Funding: This research was funded by the Cooperative Education Program of Ministry of Education of China (No. 201801238024), Innovation and Entrepreneurship Education Center Project of Hunan Provincial Education Department ([2018]380-74), and Chenzhou Municipal Science and Technology Bureau of Research on Real-Time Monitoring System of Intelligent Trash Can ([2018]102).

Acknowledgments: We deeply appreciate the computing resources offered by the National Supercomputing Center in Shenzhen, China.

Conflicts of Interest: The authors declare no conflict of interest.

\section{References}

1. Bibienne, T.; Tousignant, M.; Bobet, J.L.; Huot, J. Synthesis and hydrogen sorption properties of $\mathrm{TiV}_{(2-x)} \mathrm{Mn}_{x}$ BCC alloys. J. Alloy. Compd. 2015, 624, 247-250. [CrossRef] 
2. Massicot, B.; Latroche, M.; Joubert, J.M. Hydrogenation properties of Fe-Ti-V bcc alloys. J. Alloy. Compd. 2011, 509, 372-379. [CrossRef]

3. Huot, J.; Ravnsbæk, D.B.; Zhang, J.; Cuevas, F.; Latroche, M.; Jensen, T.R. Mechanochemical synthesis of hydrogen storage materials. Prog. Mater. Sci. 2013, 58, 30-75. [CrossRef]

4. Yu, X.B.; Yang, Z.X.; Feng, S.L.; Wu, Z.; Xu, N.X. Influence of Fe addition on hydrogen storage characteristics of Ti-V-based alloy. Int. J. Hydrog. Energy 2006, 31, 1176-1181. [CrossRef]

5. Iba, H.; Akiba, E. Hydrogen absorption and modulated structure in Ti-V-Mn alloys. J. Alloy. Compd. 1997, 253, 21-24. [CrossRef]

6. Yu, X.B.; Wu, Z.; Xia, B.J.; Huang, T.Z.; Chen, J.Z.; Wang, Z.S.; Xu, N.X. Hydrogen storage in Ti-V-based body-centered-cubic phase alloys. J. Mater. Res. 2003, 18, 2533-2536. [CrossRef]

7. Nomura, K.; Akiba, E. $\mathrm{H}_{2}$ Absorbing-desorbing characterization of the Ti-V-Fe alloy system. J. Alloy. Compd. 1995, 231, 513-517. [CrossRef]

8. Seo, C.Y.; Kim, J.H.; Lee, P.S.; Lee, J.Y. Hydrogen storage properties of vanadium-based b.c.c. solid solution metal hydrides. J. Alloy. Compd. 2003, 348, 252-257. [CrossRef]

9. Pan, H.G.; Li, R.; Gao, M.X.; Liu, Y.F.; Wang, Q.D. Effects of Cr on the structural and electrochemical properties of TiV-based two-phase hydrogen storage alloys. J. Alloy. Compd. 2005, 404, 669-674. [CrossRef]

10. Balcerzak, M. Structure and hydrogen storage properties of mechanically alloyed Ti-V alloys. Int. J. Hydrog. Energy 2017, 42, 23698-23707. [CrossRef]

11. Dou, T.; Wu, Z.; Mao, J.F.; Xu, N.X. Application of commercial ferrovanadium to reduce cost of Ti-V-based BCC phase hydrogen storage alloys. Mater. Sci. Eng. A 2008, 476, 34-38. [CrossRef]

12. Milman, V.; Winkler, B.; White, J.A.; Pickard, C.J.; Payne, M.C.; Akhmatskaya, E.V.; Nobes, R.H. Electronic Structure, Properties, and Phase Stability of Inorganic Crystals: A Pseudopotential Plane-Wave Study. Int. J. Quantum Chem 2000, 77, 895-910. [CrossRef]

13. Segall, M.D.; Lindan, P.J.D.; Probert, M.J.; Pickard, C.J.; Hasnip, P.J.; Clark, S.J.; Payne, M.C. First-Principles Simulation: Ideas, Illustrations and the CASTEP Code. J. Phys. Condens. Matter 2002, 14, 2717-2744. [CrossRef]

14. Clark, S.J.; Segall, M.D.; Pickard, C.J.; Hasnip, P.J.; Probert, M.I.J.; Refson, K.; Payne, M.C. First principles methods using CASTEP. Z. Krist. Cryst. Mater. 2005, 220, 567-570. [CrossRef]

15. Perdew, J.P.; Burke, K.; Ernzerhof, M. Generalized Gradient Approximation Made Simple. Phys. Rev. Lett. 1996, 77, 3865-3868. [CrossRef]

16. Vanderbilt, D. Soft self-consistent pseudopotentials in a generalized eigenvalue formalism. Phys. Rev. B 1990, 41, 7892-7895. [CrossRef]

17. Monkhorst, H.J.; Pack, J.D. Special points for Brillouin-zone integrations. Phys. Rev. B 1976, 13, 5188-5192. [CrossRef]

18. Fischer, T.H.; Almlof, J. General methods for geometry and wave function optimization. J. Phys. Chem. 1992, 96, 9768-9774. [CrossRef]

19. Birch, F. Finite Elastic Strain of Cubic Crystals. Phys. Rev. 1947, 71, 809-824. [CrossRef]

20. Aurelio, G.; Guillermet, A.F.; Cuello, G.J.; Campo, J. Metastable phases in the Ti-V system: Part I. Neutron diffraction study and assessment of structural properties. Metall. Mater. Trans. A 2002, 33, 1307-1317. [CrossRef]

21. Ming, L.C.; Manghnani, M.H.; Katahara, K.W. Phase transformations in the Ti-V system under high pressure up to $25 \mathrm{GPa}$. Acta Metall. 1981, 29, 479-485. [CrossRef]

22. Basak, S.; Shashikala, K.; Sengupta, P.; Kulshreshtha, S.K. Hydrogen absorption properties of Ti-V-Fe alloys: Effect of Cr substitution. Int. J. Hydrog. Energy 2007, 32, 4973-4977. [CrossRef]

23. Hagi, T.; Sato, Y.; Yasuda, M.; Tanaka, K. Structure and phase diagram of the Ti-V-H system at room temperature. Trans. Jpn. Inst. Met. 1987, 28, 198-204. [CrossRef]

24. Stern, A.; Kaplan, N.; Shaltiel, D. Superconducting transition temperatures of the system $\mathrm{V}_{1-x} \mathrm{Ti}_{x} \mathrm{H}_{y}$. Solid State Commun. 1981, 38, 445-450. [CrossRef]

25. Ikehata, H.; Nagasako, N.; Furuta, T.; Fukumoto, A.; Miwa, K.; Saito, T. First-principles calculations for development of low elastic modulus Ti alloys. Phys. Rev. B 2004, 70, 174113. [CrossRef]

26. Wang, J.H.; Yip, S.; Phillpot, S.R.; Wolf, D. Crystal instabilities at finite strain. Phys. Rev. Lett. 1993, 71, 4182-4185. [CrossRef] 
27. Patil, S.K.R.; Khare, S.V.; Tuttle, B.R.; Bording, J.K.; Kodambaka, S. Mechanical stability of possible structures of PtN investigated using first-principles calculations. Phys. Rev. B 2006, 73, 104118. [CrossRef]

28. Pugh, S.F. XCII. Relations between the elastic moduli and the plastic properties of polycrystalline pure metals. Philos. Mag. 1954, 45, 823-843. [CrossRef]

29. Cao, Y.; Zhu, J.C.; Nong, Z.S.; Yang, X.W.; Liu, Y.; Lai, Z.H. First-principles studies of the structural, elastic, electronic and thermal properties of $\mathrm{Ni}_{3} \mathrm{Nb}$. Comput. Mater. Sci. 2013, 77, 208-213. [CrossRef]

30. Iotova, D.; Kioussis, N.; Lim, S.P. Electronic structure and elastic properties of the $\mathrm{Ni}_{3} X(X=\mathrm{Mn}, \mathrm{Al}, \mathrm{Ga}, \mathrm{Si}$, Ge) intermetallics. Phys. Rev. B 1996, 54, 14413-14422. [CrossRef]

31. Hill, R. The elastic behaviour of a crystalline aggregate. Proc. Phys. Soc. Sect A 1952, 65, 349-354. [CrossRef]

32. Mattesini, M.; Ahuja, R.; Johansson, B. Cubic $\mathrm{Hf}_{3} \mathrm{~N}_{4}$ and $\mathrm{Zr}_{3} \mathrm{~N}_{4}$ : A class of hard materials. Phys. Rev. B 2003, 68, 184108. [CrossRef]

33. Fu, H.Z.; Li, D.H.; Peng, F.; Gao, T.; Cheng, X.L. Ab initio calculations of elastic constants and thermodynamic properties of NiAl under high pressures. Comput. Mater. Sci. 2008, 44, 774-778. [CrossRef]

34. Yoo, M.H. On the theory of anomalous yield behavior of $\mathrm{Ni}_{3} \mathrm{Al}$-Effect of elastic anisotropy. Scr. Metall. 1986, 20, 915-920. [CrossRef]

35. Lau, K.; Mccurdy, A.K. Elastic anisotropy factors for orthorhombic, tetragonal, and hexagonal crystals. Phys. Rev. B 1998, 58, 8980-8984. [CrossRef]

36. Fu, H.Z.; Li, X.F.; Liu, W.F.; Ma, Y.M.; Gao, T.; Hong, X.H. Electronic and dynamical properties of NiAl studied from first principles. Intermetallics 2011, 19, 1959-1967. [CrossRef]

37. Reed, R.P.; Clark, A.F. American Society of Metals; Metals Park: Geauga County, OH, USA, 1983.

38. Friák, M.; Šob, M.; Vitek, V. Ab initio calculation of tensile strength in iron. Philos. Mag. 2003, 83, 3529-3537. [CrossRef]

39. Fu, H.Z.; Peng, W.M.; Gao, T. Structural and elastic properties of ZrC under high pressure. Mater. Chem. Phys. 2009, 115, 789-794. [CrossRef]

40. Pettifor, D.G. Theoretical predictions of structure and related properties of intermetallics. Mater. Sci. Technol. 1992, 8, 345-349. [CrossRef]

41. Johnson, R.A. Analytic nearest-neighbor model for fcc metals. Phys. Rev. B 1988, 37, 3924-3931. [CrossRef]

(C) 2019 by the authors. Licensee MDPI, Basel, Switzerland. This article is an open access article distributed under the terms and conditions of the Creative Commons Attribution (CC BY) license (http://creativecommons.org/licenses/by/4.0/). 